\title{
Nonreciprocal and even Willis couplings in periodic thermoacoustic amplifiers
}

\author{
Côme Olivier $\odot$, Gaëlle Poignand $\odot$, Matthieu Malléjac $\odot$, Vicent Romero-García $\odot$, and Guillaume Penelet \\ Laboratoire d'Acoustique de l'Université du Mans (LAUM), UMR 6613, Institut d'Acoustique - Graduate School (IA-GS), \\ CNRS, Le Mans Université, France \\ Aurélien Merkel \\ Université de Lorraine, CNRS, Institut Jean Lamour, F-54000 Nancy, France \\ Daniel Torrent \\ GROC, UJI, Institut de Noves Tecnologies de la Imatge (INIT), Universitat Jaume I, 12071 Castelló, Spain \\ Jensen Li \\ Department of Physics, The Hong Kong University of Science and Technology, Clear Water Bay, Hong Kong, China \\ Johan Christensen \\ Department of Physics, Universidad Carlos III de Madrid, 28911 Leganés, Madrid, Spain \\ Jean-Philippe Groby* \\ Laboratoire d'Acoustique de l'Université du Mans (LAUM), UMR 6613, Institut d'Acoustique - Graduate School (IA-GS), \\ CNRS, Le Mans Université, France
}

(Received 20 May 2021; revised 29 September 2021; accepted 27 October 2021; published 10 November 2021)

\begin{abstract}
Thermoacoustic amplifiers are analyzed in the framework of nonreciprocal Willis coupling. The closed form expressions of the effective properties are derived, showing that an applied temperature gradient causes the appearance of a nonreciprocal Willis coupling. Even and nonreciprocal Willis couplings are exhibited already in the first-order Taylor expansion of the solution and are of equal modulus but opposite sign, thus suggesting that the even Willis coupling is a reaction to the nonreciprocity introduced by the temperature gradients. These Willis couplings cause a coalescence point in the $k$ space, which deviates from $\operatorname{Re}(k)=0$ (with $k$ the wave number) and is thus a zero-group-velocity point, as well as the opening of an amplification gap at low frequency. Effective parameters and scattering properties are found in excellent agreement with experimental results. This article paves the way to further control the acoustic waves at very low frequencies with nonreciprocal systems.
\end{abstract}

DOI: 10.1103/PhysRevB.104.184109

\section{INTRODUCTION}

Swapping source and receiver has long provided identical frequency responses due to reciprocity features, thus preventing the possible tuning of the transmission coefficient in opposite directions. In acoustics and elasticity, the reciprocity of wave propagation can be broken via three means: by using spatiotemporal dependent material properties [1], by combining nonlinear properties with an asymmetry [2-4], or by introducing an external bias [5,6]. Nonreciprocal devices and their applications have recently been reviewed in Ref. [7]. Among the various possibilities offered by the introduction of an external bias in acoustic systems, thermoacoustics, although effective [8,9], is often neglected. Thermoacoustics is concerned with the thermal interaction between an acoustic wave and the surrounding waveguide wall $[10,11]$. Although research in thermoacoustics has mostly been

\footnotetext{
*jean-philippe.groby@univ-lemans.fr
}

oriented towards the development of thermodynamic machines and the understanding of the phenomena controlling their behavior - on the one hand, the thermoacoustic prime movers (or engines) [12,13]; on the other hand, thermoacoustic heat pumps and refrigerators $[14,15]$ - more fundamental research has relied on the thermoacoustic amplification or damping of acoustic waves in recent years. Quasiperiodic and chaotic oscillations $[16,17]$ or synchronization phenomena involved in thermally driven autonomous oscillators $[18,19]$, thermoacoustic shock waves [20,21], and solitary waves [22], thermoacoustic diode [8], or a $\mathcal{P} \mathcal{T}$-symmetric system [23] have been the focus of a growing interest. Thermoacoustics still appears to be overlooked as far as nonreciprocal systems are concerned.

Meanwhile, Willis materials have received an increasing interest since the publication of the seminal work [24], thanks to their analogy with bi-isotropic electromagnetic metamaterials [25]. The Willis coupling parameters couple the potential and kinetic energies in the acoustic conservation relations, therefore enhancing the ability to control waves in these 
metamaterials compared to other materials that do not exhibit such coupling. Three categories of Willis coupling parameters have been highlighted: the even coupling related to the structure asymmetry, the odd coupling related to nonlocal effect (although its existence is questioned for one-dimensional systems), and the nonreciprocal coupling [26]. Only a few nonreciprocal systems [1,27-29] have been modeled and analyzed as Willis materials so far. Interestingly, nonreciprocal Willis coupling was found to be nonzero already in the firstorder Taylor expansion of the solution, while even Willis coupling was found to be nonzero in the second-order Taylor expansion of the solution. None of these articles derived closed form expressions of the coupling parameters and investigated the possible induced asymmetry by the nonreciprocity.

In this article we derive the closed form expressions of the effective properties of a one-dimensional periodic arrangement of a thermoacoustic amplifier following Ref. [30]. The procedure relies on the Padé's approximation of the total transfer matrix that links the state vectors at both sides of the unit cell and directly provides both even and nonreciprocal Willis coupling terms. The analysis of the effective parameters as well as the dispersion relation show that the system possesses a coalescence point in the $k$ space [31] that resembles a $\mathcal{P} \mathcal{T}$-broken phase and yields to zero-group velocity for given temperature differences.

\section{GENERAL STATEMENT}

\section{A. Wave propagation in a duct submitted to a temperature gradient}

Assuming an implicit time dependence $e^{-i \omega t}$ and following Refs. [10,32], the equations that describe the acoustic wave propagation in a duct of cross-sectional area $S$ in the presence of a longitudinal temperature gradient $\partial T_{m} / \partial x$ are

$$
\begin{aligned}
\frac{\partial p}{\partial x} & =\frac{i \omega \rho_{m}}{S \phi\left(1-f_{v}\right)} \mathscr{V}, \\
\frac{\partial \mathscr{V}}{\partial x} & =\frac{i \omega S \phi}{\gamma p_{m}}\left[1+(\gamma-1) f_{\kappa}\right] p+\frac{f_{\kappa}-f_{v}}{\left(1-f_{v}\right)(1-\operatorname{Pr})} \frac{1}{T_{m}} \frac{\partial T_{m}}{\partial x} \mathscr{V},
\end{aligned}
$$

where $p$ is the acoustic pressure, $\mathscr{V}=S \phi V$ is the volume flow rate, with $V$ the particle velocity and $\phi$ the porosity across the waveguide, $\rho_{m}, p_{m}$, and $T_{m}$ are the mean-state values (across the section) of the density, pressure (atmospheric pressure), and temperature respectively, $f_{\nu}$ and $f_{\kappa}$ are complex frequency-dependent functions, which account respectively for viscous and thermal losses [10] (see also Appendix A), $\gamma$ is the specific heat ratio, and $\operatorname{Pr}$ is the Prandtl number. Introducing the state vector $\mathbf{W}=\langle p, \mathscr{V}\rangle^{T}$, where $T$ is the transpose operator, these equations can thus be cast in the matrix form

$$
\frac{\partial}{\partial x} \mathbf{W}=\left[\begin{array}{cc}
0 & i \omega \rho(x) \\
i \omega C(x) & \mathscr{G}(x)
\end{array}\right] \mathbf{W}=\mathbf{A}(x) \mathbf{W},
$$

where $\rho(x)$ is the effective density, $C(x)$ is the effective compressibility, i.e., the inverse of the effective bulk modulus $K(x)$, and $\mathscr{G}(x)$ is a "gain" term depending on the temperature gradient $\partial T_{m} / \partial x$ that represents the thermoacoustic amplification/attenuation. The choice of the volume flow rate as a second component of the state vector instead of the particle velocity is motivated by the continuity conditions (continuity of pressure and volume flow rate) at interfaces between different materials and ducts of different cross sections. Note that the effective bulk modulus and density are thus both divided by the waveguide cross section because of the use of the volume flow rate as the second component of the state vector. Thus, the effective bulk modulus and density are bulk modulus per unit area (given in $\mathrm{Pa} \mathrm{m}^{-2}$ ) and density per unit area (given in $\mathrm{Kg} \mathrm{m}^{-5}$ ). Note in addition that the effective bulk modulus and density are spatially dependent in an implicit way (but obviously frequency dependent), notably because the fluid constants that appear in both functions $f_{\nu}$ and $f_{\kappa}$ depend on the temperature distribution $T_{m}(x)$. The propagation matrix A depending on $x$ and not commuting for different values of $x$, the solution of the system Eq. (2), which relates the state vector at a position $l$ to that at a position 0 via $\mathbf{W}(l)=\mathbf{M}_{l} \mathbf{W}(0)$ involves a matricant $\mathbf{M}_{l}$ that is evaluated through a Peano series expansion [33]

$$
\mathbf{M}_{l}=\mathbf{I d}+\int_{0}^{l} \mathbf{A}(x) d x+\int_{0}^{l} \mathbf{A}(x)\left(\int_{0}^{x} \mathbf{A}(\zeta) d \zeta\right) d x+\cdots
$$

Of particular interest is the first-order expansion, which reads as

$$
\mathbf{M}_{l}^{(1)}=\mathbf{I d}+\int_{0}^{l} \mathbf{A}(x) d x=\left[\begin{array}{cc}
1 & i \omega \bar{\rho} l \\
i \omega \bar{C} l & 1+\overline{\mathscr{G}} l
\end{array}\right],
$$

where $\bar{\rho}, \bar{C}$, and $\overline{\mathscr{G}}$ are the mean values of $\rho(x), C(x)$, and $\mathscr{G}(x)$ over the length $l$, i.e., $\bar{\cdot}=\frac{1}{l} \int_{0}^{l} d x$. This first-order iteration corresponds to a first-order Taylor expansion of $\exp (\mathbf{A} l)$, when $\mathbf{A}$ does not depend on $x$. Introducing $\bar{k}=\omega \sqrt{\bar{\rho} \bar{C}}$, the determinant of $\mathbf{M}_{l}^{(1)}$ reads in the first-order expansion

$$
\operatorname{det}\left(\mathbf{M}_{l}^{(1)}\right)=1+\overline{\mathscr{G}} l+O\left[(\bar{k} l)^{2}\right],
$$

which clearly shows that the system is not reciprocal in the presence of the gain term, i.e., when $\partial T_{m} / \partial x \neq 0$. The term $\overline{\mathscr{G}} l$ is a marker of the nonreciprocity that makes $\operatorname{det}\left(\mathbf{M}_{l}^{(1)}\right) \neq$ 1. When $\overline{\mathscr{G}}=0$ (i.e., $\partial T_{m} / \partial x=0$ ), the system falls back to reciprocal with $\operatorname{det}\left(\mathbf{M}_{l}^{(1)}\right)=1$. The term $\mathcal{G}=1+\overline{\mathscr{G}} l$ is thus called nonreciprocity term in the following. In reality, Eq. (4) relies on two assumptions and is $O\left[(\bar{k} l)^{2}\right]$, i.e., the system length is much smaller than the wavelength, but also $O\left[(\overline{\mathscr{G}} l)^{2}\right]$, i.e., the gain per unit length is weak. Effectively, the dimensionless term $\overline{\mathscr{G}} l$ mainly depends on the temperature gradient and is thus driven independently of $\bar{k} l$. Note that Eq. (5) turns out to be $O[(\bar{k} l)]$ but $O\left[(\overline{\mathscr{G}} l)^{2}\right]$. The determinant does not depend on $\bar{k} l$ and only depends on $\overline{\mathscr{G}} l$, thus emphasizing the central role of $\overline{\mathscr{G}} l$ in the system nonreciprocity.

\section{B. Calculation of the effective properties from the knowledge of the transfer matrix of the unit cell}

We assume a one-dimensional nonreciprocal and asymmetric system composed of a $d$-periodic repetition of a unit cell of respective propagation matrix $\mathbf{A}_{e}$. The system is homogenized and $\mathbf{A}_{e}$ does not depend on $x$. The state vectors at both sides of the unit cell $\mathbf{W}(d)$ and $\mathbf{W}(0)$ are related to each other through the $2 \times 2$ transfer matrix $\mathbf{T}=\exp \left(\mathbf{A}_{e} d\right)$ of elements $t_{i j},(i, j)$ 


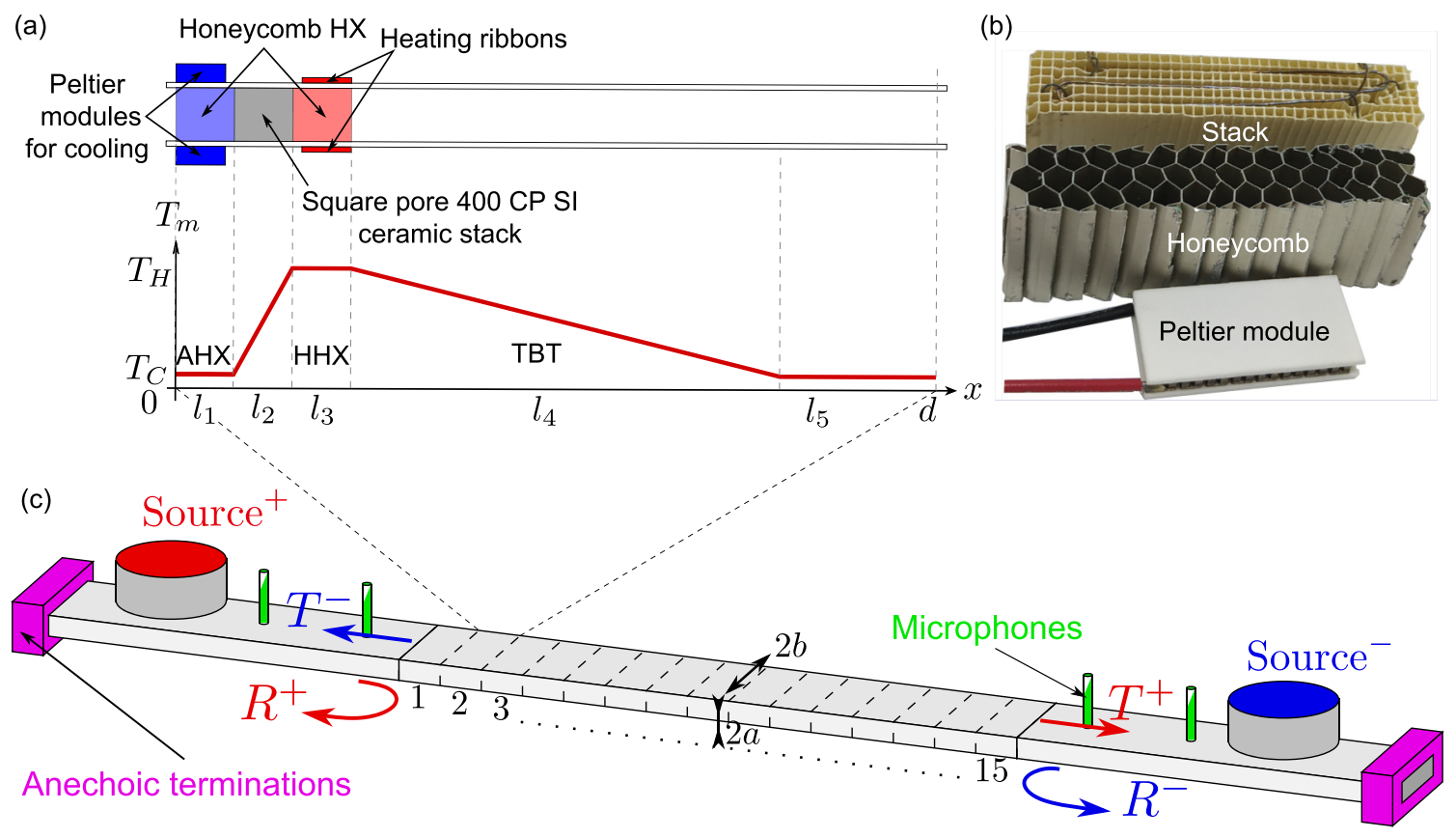

FIG. 1. (a) Scaled representation of the unit cell together with the temperature distribution along the unit cell. The length of the experimental cell is $d=195 \mathrm{~mm}$. (b) Photography of the elements of the thermoacoustic cell and (c) schematic view of the experimental setup for measuring the scattering matrix of the thermoacoustic amplifier.

$\in[1,2]$. Following Ref. [30] the propagation matrix is correctly approximated by the inversion of the first-order Padé's approximation of the matrix exponential,

$$
\mathbf{A}_{e} \approx \frac{2}{d}(\mathbf{T}+\mathbf{I d})^{-1}(\mathbf{T}-\mathbf{I d}) \approx \frac{2}{d} \frac{1}{\operatorname{Tr}(\mathbf{T})+\operatorname{det}(\mathbf{T})+1}\left[\begin{array}{cc}
t_{11}-t_{22}+\operatorname{det}(\mathbf{T})-1 & 2 t_{12} \\
2 t_{21} & t_{22}-t_{11}+\operatorname{det}(\mathbf{T})-1
\end{array}\right],
$$

with $\operatorname{Tr}(\mathbf{T})=t_{11}+t_{22}$. Equation (6) directly provides the elements of a nonreciprocal Willis material

$$
\mathbf{A}_{e}=i \omega\left[\begin{array}{cc}
\chi_{e}^{a}+\chi_{e}^{\mathrm{nr}} & \rho_{e} \\
C_{e} & -\chi_{e}^{a}+\chi_{e}^{\mathrm{nr}}
\end{array}\right]
$$

where $\rho_{e}$ is the effective density, $C_{e}$ is the effective compressibility, $\chi_{e}^{a}$ is the even Willis coupling, and $\chi_{e}^{\mathrm{nr}}$ is the nonreciprocal Willis coupling, respectively, equal to

$$
\begin{array}{ll}
\rho_{e}=\frac{-4 i}{\omega d} \frac{t_{12}}{1+\operatorname{Tr}(\mathbf{T})+\operatorname{det}(\mathbf{T})}, & C_{e}=\frac{-4 i}{\omega d} \frac{t_{21}}{1+\operatorname{Tr}(\mathbf{T})+\operatorname{det}(\mathbf{T})}, \\
\chi_{e}^{a}=\frac{-2 i}{\omega d} \frac{t_{11}-t_{22}}{1+\operatorname{Tr}(\mathbf{T})+\operatorname{det}(\mathbf{T})}, \quad \chi_{e}^{\mathrm{nr}}=\frac{-2 i}{\omega d} \frac{\operatorname{det}(\mathbf{T})-1}{1+\operatorname{Tr}(\mathbf{T})+\operatorname{det}(\mathbf{T})} .
\end{array}
$$

When considering reciprocal systems, $\operatorname{det}(\mathbf{T})=1$ and thus $\chi_{e}^{\mathrm{nr}}=0$. The expressions of the effective parameters then reduce to those provided in Ref. [30]. In addition, for symmetric systems $t_{11}=t_{22}$ and as a consequence $\chi_{e}^{a}=0$.

\section{EXPRESSION OF THE EFFECTIVE PROPERTIES OF A PERIODIC THERMOACOUSTIC AMPLIFIER}

We consider a $d$-periodic thermoacoustic amplifier, the unit cell of which consists of five elements in series of respective lengths $l_{j}, j=1, \ldots, 5$, such that $d=\sum_{j=1}^{5} l_{j}$, see Fig. 1(a). The $l_{2}$-long porous material, where the thermoacoustic effect takes place (customarily called stack), is subjected to a temperature gradient applied with the help of an ambient and hot heat exchangers (denoted AHX and HHX) of respective lengths $l_{1}$ and $l_{3}$. The heat exchangers impose a temperature difference $\Delta T=T_{H}-T_{C}$, between the hot $T_{H}$ and the ambient $T_{C}$ temperatures. The HHX is followed by an empty tube of total length $l_{4}+l_{5}$. The temperature distribution loops back to the temperature of the AHX along the $l_{4}$-long portion, which is therefore called the thermal buffer tube (TBT). In this study, the resulting temperature distribution is considered one dimensional, homogeneous in the heat exchangers, and varies linearly in the stack and the TBT, as depicted in Fig. 1(a). The temperature gradient $\partial T_{m} / \partial x$ is thus positive along the stack, while it is negative along the TBT, here also with reference to Fig. 1(a). No temperature gradient is applied on the elements 1,3 , and 5 . These elements are thus reciprocal. 
The state vector at the end of the unit cell $\mathbf{W}(d)$ is related to that at the other end $\mathbf{W}(0)$ via the full transfer matrix $\mathbf{T}$ which is the multiplication of the five matrices modeling the propagation along the five elements of the unit cell

$$
\mathbf{W}(d)=\mathbf{T W}(0)=\mathbf{T}_{l_{5}} \mathbf{M}_{l_{4}}^{(1)} \mathbf{T}_{l_{3}} \mathbf{M}_{l_{2}}^{(1)} \mathbf{T}_{l_{1}} \mathbf{W}(0),
$$

where $\mathbf{T}_{l}=\exp (\mathbf{A} l)$ is the usual transfer matrix along a distance $l$ with $\mathbf{A}$ the propagation matrix of the element, the first-order Taylor expansion of which takes the form

$\mathbf{T}_{l}=\left[\begin{array}{cc}\cos (k l) & i Z \sin (k l) \\ i \sin (k l) / Z & \cos (k l)\end{array}\right] \approx\left[\begin{array}{cc}1 & i \omega \rho l \\ i \omega C l & 1\end{array}\right]+O\left[(k l)^{2}\right]$,

where $k$ is the wave number and $Z$ is the impedance (divided by the duct cross-sectional area in our case). Equation (10) formally corresponds to Eq. (4) in the absence of gain term. Viscothermal losses are accounted for in each element, see Appendix A. Considering the first-order Taylor expansion of the total transfer matrix elements, we end up with

$$
\begin{aligned}
\rho_{e} & =\frac{2}{d\left[1+\mathcal{G}_{2} \mathcal{G}_{4}\right]}\left(\rho_{1} l_{1}+\bar{\rho}_{2} l_{2}+\left[\rho_{3} l_{3}+\bar{\rho}_{4} l_{4}+\rho_{5} l_{5} \mathcal{G}_{4}\right] \mathcal{G}_{2}\right), \\
C_{e} & =\frac{2}{d\left[1+\mathcal{G}_{2} \mathcal{G}_{4}\right]}\left(C_{5} l_{5}+\bar{C}_{4} l_{4}+\left[C_{3} l_{3}+\bar{C}_{2} l_{2}+C_{1} l_{1} \mathcal{G}_{2}\right] \mathcal{G}_{4}\right), \\
\chi_{e}^{a} & =\frac{-i\left[1-\mathcal{G}_{2} \mathcal{G}_{4}\right]}{d \omega\left[1+\mathcal{G}_{2} \mathcal{G}_{4}\right]}, \\
\chi_{e}^{\mathrm{nr}} & =\frac{i\left[1-\mathcal{G}_{2} \mathcal{G}_{4}\right]}{d \omega\left[1+\mathcal{G}_{2} \mathcal{G}_{4}\right]},
\end{aligned}
$$

where all the effective parameters are in $O\left[(k d)^{2}\right]$.

This result calls for several comments.

First, all the effective parameters are impacted by the nonreciprocal terms, notably via a common denominator $1+\mathcal{G}_{2} \mathcal{G}_{4}$. This denominator arises from the assumption that both $\overline{\mathscr{G}}_{2}$ and $\overline{\mathscr{G}}_{4}$ are $O[(k d)]$. This implies that $\rho_{e} S / \rho_{0}<1$ and $K_{e} S / \gamma P_{0}<1$ at low frequencies, where $\rho_{0}$ and $P_{0}$ are, respectively, the ambient (air) density and atmospheric pressure. Please note that Eqs. (11) also rely on the assumption that Eq. (4) is $O\left[(\overline{\mathscr{G}} d)^{2}\right]$. Thus, $\mathcal{G}_{2} \mathcal{G}_{4}=1+\overline{\mathscr{G}}_{2} l_{2}+\overline{\mathscr{G}}_{4} l_{4}+$ $O\left[(\overline{\mathscr{G}} d)^{2}\right]$ rigorously.

Second, the thermoacoustic amplifier appears as a Willis material and exhibits both even and nonreciprocal coupling parameters at the first-order Taylor expansion. This constitutes a major difference with respect to laminate or resonant Willis materials, which require second-order Taylor expansion to exhibit Willis coupling [26,30].

Third, $\chi_{e}^{a}=-\chi_{e}^{\mathrm{nr}}$, which implies that the top left element of the effective propagation matrix is null in the first-order expansion. The propagation matrix thus strongly resembles Eq. (2). This suggests that the thermoacoustics' fundamental equations Eq. (1) are a specific form of those of a nonreciprocal Willis material. Together with the second comment, we conclude that the even Willis coupling arises from the asymmetry of the temperature gradient and appears in reaction to the nonreciprocity. This becomes clear when both temperature gradients are of opposite sign so as to lead to $\overline{\mathscr{G}}_{2} l_{2}=-\overline{\mathscr{G}}_{4} l_{4}$. In this case, $\mathcal{G}_{2} \mathcal{G}_{4}=1+O\left[\left(\overline{\mathscr{G}}_{d}\right)^{2}\right]$ and thus $\chi_{e}^{a}=-\chi_{e}^{\mathrm{nr}}=0$ in the first-order expansion, while the system material organization is asymmetric. Both Willis coupling parameters only depend on the temperature gradient and not on the material asymmetry in the first-order expansion.

Fourth, when $\overline{\mathscr{G}}_{2}$ and $\overline{\mathscr{G}}_{4}$ are both equal to zero, the effective parameters collapse to those of a usual laminate fluid in the first-order expansion, with $\chi_{e}^{a}=\chi_{e}^{\mathrm{nr}}=0$. Note that the Willis even coupling term does not vanish in that case when second-order expansions are used because the unit cell involves different materials and is asymmetric [30].

Fifth, the form of both $\rho_{e}$ and $C_{e}$ exhibits different cumulative impacts of the temperature gradients. While the temperature gradient impacts the following density terms, i.e., the density terms following a nonreciprocal element are multiplied by the product of all the preceding nonreciprocity terms, it impacts the preceding compressibility terms, i.e., the effective compressibility terms preceding a nonreciprocal element are multiplied by all the following nonreciprocity terms. The effect of the temperature gradient on the density derives directly from its flow source nature. Its effect on the compressibility is more complex and can be analyzed in the way that the temperature gradient acts as a volume flow amplifier rather than a pure flow source, with the volume flow rate depending on the previous compressibilities.

Sixth, both the even and nonreciprocal coupling parameters are almost purely imaginary and nonzero at low frequency for a thermoacoustic amplifier. The dispersion relation thus exhibits at first glance a band gap at zero frequency that is shifted along the imaginary wave-number axis, i.e., $k_{e}^{ \pm}=$ $-i \omega\left|\chi_{e}^{\mathrm{nr}}\right| \pm \omega \sqrt{-\left|\chi_{e}^{a}\right|^{2}+\rho_{e} C_{e}}$, see Appendix B.

\section{EXPERIMENTAL AND NUMERICAL VALIDATIONS OF THE EFFECTIVE PARAMETERS AND DISCUSSION}

The experimental setup consists of a waveguide of rectangular cross section $(2 a \times 2 b=1 \mathrm{~cm} \times 5 \mathrm{~cm})$ containing either a single thermoacoustic cell or 15 identical thermoacoustic cells of length $d=19.5 \mathrm{~cm}$ in both cases, as depicted in Figs. 1(a)-1(c). Only the results for the single-unit cell are discussed in this section, while those for the 15 identical cells are discussed in Appendix D. The stack is a cordierite substrate with a pore density of $400 \mathrm{CPSI}$, of length $l_{2}=15 \mathrm{~mm}$. Its pores are square of half-width $r_{s}=585 \mu \mathrm{m}$ and its porosity is $\phi=0.85$. The ambient heat exchanger is made of a section of $1 / 8 \mathrm{in}$. aluminum honeycomb (porosity $\phi=0.945$ ), with $l_{1}=15 \mathrm{~mm}$, used to ensure a uniform temperature distribution over the cross section. The hot heat exchanger is a section of the stack material of length $l_{3}=3 \mathrm{~mm}$ supporting a resistive wire providing sufficient heating power to maintain temperature up to $T_{H}=373 \mathrm{~K}$. The TBT is assumed to be of length $l_{4}=23 \mathrm{~mm}$. The thermoacoustic system is placed between two waveguides of the same cross section for the measurement of the scattering matrix by means of the twosource method [34]. As illustrated in Fig. 1, each of these measurement sections consists of a straight $1.2 \mathrm{~m} \mathrm{duct,} \mathrm{on}$ which a pair of 1/4 in. microphones are flush mounted, a moving coil loudspeaker is side mounted in a small enclosure, and ends with an anechoic termination. Each microphone pair is separated by a distance of $65 \mathrm{~cm}$, allowing measurements in the 10 to $200 \mathrm{~Hz}$ range, provided that they have been precisely calibrated (both in amplitude and phase) to limit the errors on the measured and calculated quantities [35]. A 

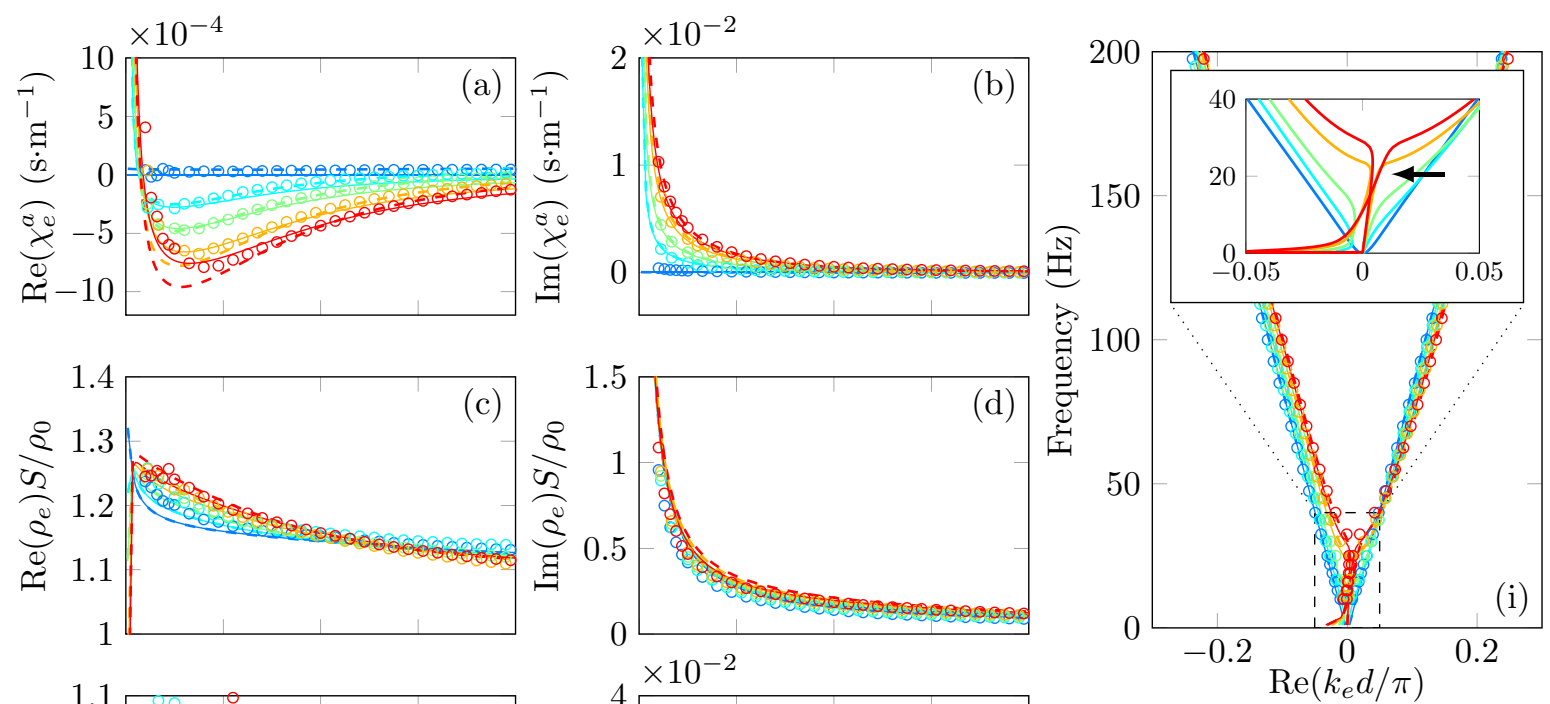

d)
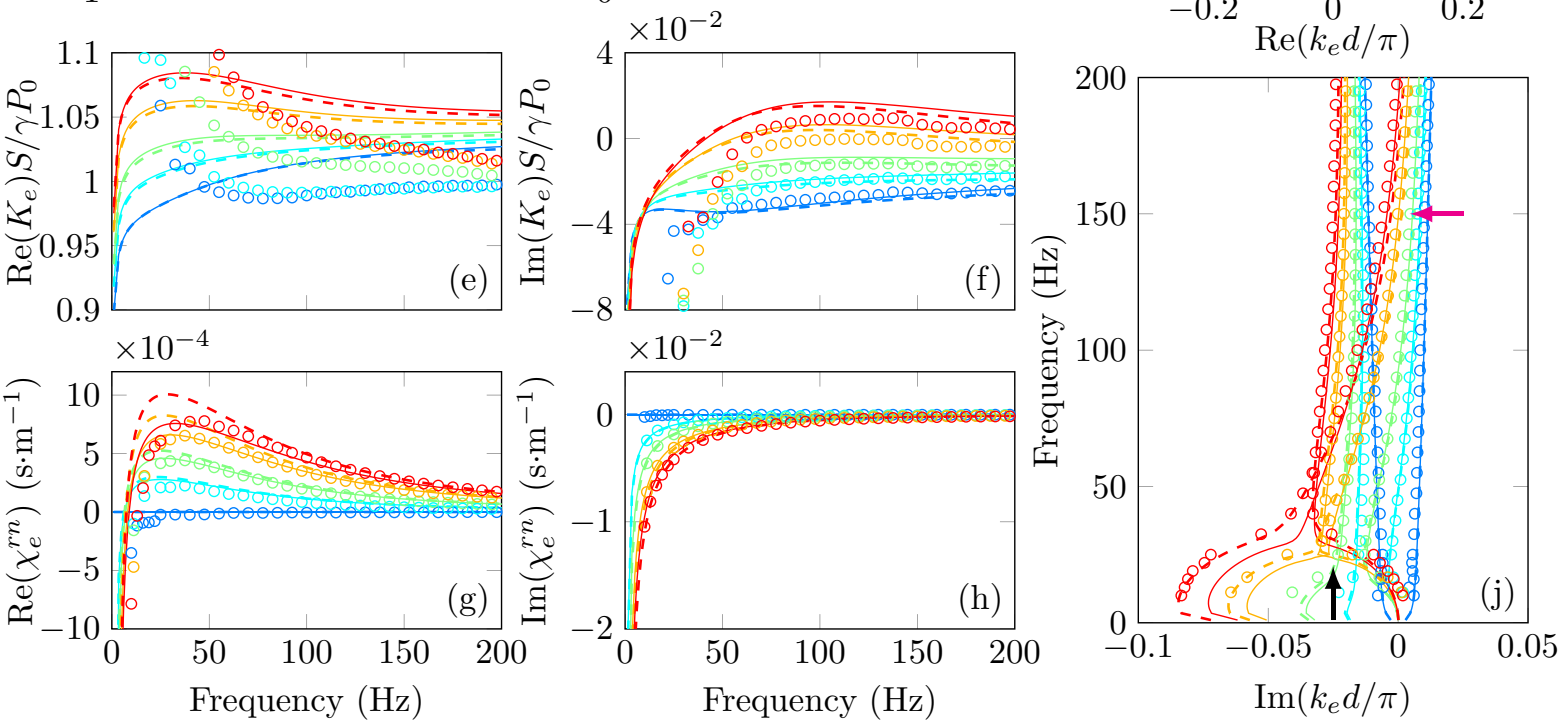

$$
\circ \Delta T=0 \mathrm{~K}, \circ \Delta T=22 \mathrm{~K}, \circ \Delta T=42 \mathrm{~K}, \circ \Delta T=75 \mathrm{~K}, \circ \Delta T=100 \mathrm{~K}
$$

FIG. 2. Real and imaginary parts of the even Willis coupling parameter (a) and (b), normalized density (c) and (d), normalized bulk modulus (e) and (f), and nonreciprocal Willis coupling parameter (g) and (h) for $\Delta T=0 \mathrm{~K}$ (blue curves), $\Delta T=22 \mathrm{~K}$ (cyan curves), $\Delta T=42 \mathrm{~K}$ (green curves), $\Delta T=75 \mathrm{~K}$ (orange curves), and $\Delta T=100 \mathrm{~K}$ (red curves). Real and imaginary parts of the dispersion relations (i) and (j). The inset of (i) shows a zoom on the real part of the dispersion relation at low frequency. The results depicted with solid curves are calculated with the expressions given in Eq. (11), the dashed curves represent the numerical evaluation with the transfer matrix method, and the circles depict the measurements.

relative calibration is therefore realized using a homemade small cavity coupler. The low frequency anechoic terminations attached to the measurement sections consist of a larger cavity $(1.5 \mathrm{~cm} \times 7 \mathrm{~cm})$ with an adjustable length closed by stainless steel wire mesh screens [36]. The reflection coefficient of such terminations has an amplitude lower than 5\% in the frequency range of interest, which is a sufficiently low value for the needs of this study. The sources are driven at amplitudes that generate sufficiently low acoustic pressures to ensure a linear system behavior during all measurements.

Figures 2(a)-2(h) depict the real and imaginary parts of the even and nonreciprocal Willis coupling parameters, normalized density, and bulk modulus as evaluated from the expressions given in Eq. (11) (solid curves), from the direct calculation with the transfer matrix method (see Appendix C and indexed by num) (dashed curves), and as measured (see Appendix B) (open circles) for $\Delta T=0 \mathrm{~K}$ (blue curves), $\Delta T=22 \mathrm{~K}$ (cyan curves), $\Delta T=42 \mathrm{~K}$ (green curves), $\Delta T=$ $75 \mathrm{~K}$ (orange curves), and $\Delta T=100 \mathrm{~K}$ (red curves). Note that the direct calculation with the transfer matrix method is conducted by discretizing the stack and the TBT (subjected to temperature gradients) in piecewise constant elements. All curves are found in good agreement. Slight discrepancies are nevertheless noticed, notably for the normalized bulk modulus [see Fig. 2(e)], which are attributed to the assumption of the linear variation of the temperature distribution, but also to the measurement difficulties at very low frequency. As pointed out in the previous section, the two Willis couplings are almost purely imaginary at low frequencies and are of equal modulus but opposite sign. Interestingly, the 

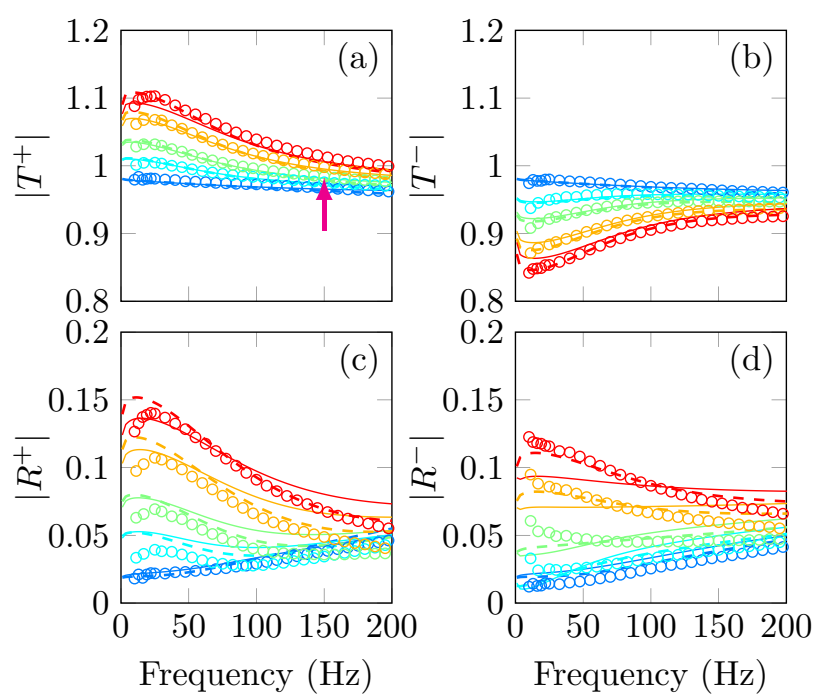

$$
\begin{gathered}
\circ \Delta T=0 \mathrm{~K}, \quad \circ \Delta T=22 \mathrm{~K}, \quad \circ \Delta T=42 \mathrm{~K}, \\
\circ \Delta T=75 \mathrm{~K}, \quad \circ \Delta T=100 \mathrm{~K}
\end{gathered}
$$

FIG. 3. Transmission and reflection coefficients along the amplification, $T^{+}$and $R^{+}$(a) and (c), and attenuation, $T^{-}$and $R^{-}$(b) and (d), directions, for $\Delta T=0 \mathrm{~K}$ (blue curves), $\Delta T=22 \mathrm{~K}$ (cyan curves), $\Delta T=42 \mathrm{~K}$ (green curves), $\Delta T=75 \mathrm{~K}$ (orange curves), and $\Delta T=100 \mathrm{~K}$ (red curves). The results as calculated with the expressions given in Eq. (11) are depicted with solid curves, as numerically evaluated from the transfer matrix method are depicted with dashed curves, and as measured are depicted with the curves marked with circles.

Willis coupling parameters do not vanish at low frequency as it is the case for laminate structures or detuned Helmholtz resonators [30]. When $\Delta T=0 \mathrm{~K}, \chi_{e}^{\mathrm{nr}}=\chi_{e}^{a}=\chi_{\mathrm{num}}^{\mathrm{nr}}=0$, but $\chi_{\text {num }}^{a} \neq 0$ although very small. This comes from the fact that $\chi_{\text {num }}^{a}$ captures the second-order expansion element which accounts for the material asymmetry [30]. While the numerically evaluated effective parameters are in good agreement with the measured parameters whatever the temperature gradient, the parameters given in Eq. (11) slightly deviate from both numerical and experimental results with increased temperature differences, mainly at low frequencies. This is particularly visible on the Willis coupling and is due to the use of the first-order Peano series expansion, for which the gain term is approximated by its mean value. Effectively, the only use of the mean value can become too harsh an approximation when the temperature gradient becomes large. This is a direct translation of the assumption that Eq. (4) is $O\left[(\overline{\mathscr{G}} l)^{2}\right]$. Nevertheless, the overall trend is correctly captured. This is also visible in Figs. 3(a)-3(d), which depict the absolute values of the reflection and transmission coefficients for each incidence, i.e., that where amplification occurs $T^{+}$and $R^{+}$and that where attenuation occurs $T^{-}$and $R^{-}$. Note that the transfer matrix is calculated via Padé's approximation when $A_{e}$ is used, thus validating the derivation of the expressions given in Eq. (11), i.e., $\left(\mathbf{I}-\mathbf{A}_{e} d / 2\right)^{-1}\left(\mathbf{I}+\mathbf{A}_{e} d / 2\right)$. When $\Delta T=0 \mathrm{~K}$, the structure is symmetric $R=R^{+}=R^{-}$and reciprocal $T=T^{+}=T^{-}$and is almost acoustically transparent, i.e., $|R| \approx 0$ and $|T| \approx 1$. As the temperature difference increases, $\left|R^{+}\right|$is different from
$\left|R^{-}\right|$which translates the asymmetry of the configuration and emphasizes the need for even Wills coupling parameters. Note that the even Willis coupling parameter is mainly driven by the asymmetric temperature gradient. Nevertheless, the amplitudes of both reflection coefficients are low, although increasing with the temperature difference. As a corollary, the amplitude of the transmission coefficients is quite large, the structure being almost acoustically transparent in the absence of a temperature gradient. Interestingly, the amplitude of $\left|T^{+}\right|$ is greater than unity, the larger the temperature difference. Conversely, the amplitude of $\left|T^{-}\right|$is smaller than unity, the larger the temperature difference. This is a direct indication of the nonreciprocity and of the amplification and attenuation directions. This difference highlights the need for the nonreciprocal Willis coupling parameter.

The real and imaginary parts of the dispersion relations are depicted in Figs. 2(i) and 2(j), respectively. The higher the temperature difference, the more asymmetric $k_{e}^{ \pm}$, which translates the nonreciprocal nature of the configuration. The trajectories of both $k_{e}^{ \pm}$are modified when a temperature gradient is applied. In particular, both $k_{e}^{ \pm}$are shifted in the negative $\operatorname{Im}(k)$ space at low frequencies. Thus, $k_{e}^{+}$is amplified while $k_{e}^{-}$is attenuated. When $\operatorname{Im}\left(k_{e}^{+}\right)$crosses back to the positive half $\operatorname{Im}(k)$ space, i.e., no amplification is observed anymore, $\left|T^{+}\right|$moves from values larger than 1 to values lower than 1 because the structure is almost acoustically transparent. This is highlighted by the magenta arrows, Figs. 2(j) and 3(a), when $\Delta T=75 \mathrm{~K}$, for example. A coalescence point in the $k$ space, where both $k_{e}^{ \pm}$are equal, is reached for a temperature difference around $\Delta T=75 \mathrm{~K}$ as pointed out by the black arrows in Figs. 2(i) and 2(j). A zero-group-velocity point is reached at this frequency, where both modes are amplified, i.e., $\operatorname{Re}\left(k_{e}^{ \pm}\right)>0$, while $\operatorname{Im}\left(k_{e}^{ \pm}\right)<0$ at this frequency. This is due to the almost purely imaginary nature of both Willis coupling parameters as explained in Sec. III. This zero-groupvelocity point is purely due to the even and nonreciprocal Willis coupling parameters and does not translate into the effective compressibility and density, as assumed in Ref. [9]. In practice, the even Willis coupling parameter is usually purely imaginary at low frequency, see Ref. [30] for example, and thus a band gap can be opened at zero frequency. Nevertheless, the even Willis coupling is usually nonzero in the second-order Taylor expansion for purely asymmetric laminated or resonant structures and usually vanishes notably in the case of Helmholtz resonators. This band gap is thus rarely or never noticed. The story is completely different in nonreciprocal systems, in which the even coupling is nonzero already in the first-order expansion and does not necessarily vanish at low frequencies. It is also accompanied by the nonreciprocal Willis coupling that shifts the mode in the $\operatorname{Im}(k)$ space. This behavior was already noticed at much higher frequencies and at a higher pass band in Ref. [27]. This coalescence point in the $k$ space does not seem to translate into a particular behavior of the scattering matrix, although the reflection and transmission coefficients present inflection points at a frequency that is close. Interestingly, the $k_{e}^{-}$branch presents infinite group velocity points for lower frequencies and higher temperature differences, which find translation neither in the effective parameters, nor in the scattering elements. 


\section{CONCLUSION}

A periodic one-dimensional thermoacoustic amplifier is analyzed as a Willis material. The closed form expressions of the effective properties are derived from Padé's approximation of the total transfer matrix that links the state vectors at both sides of the unit cell. A first-order Taylor expansion of the transfer matrix elements is sufficient to derive the effective properties notably both the even and nonreciprocal Willis couplings. The odd Willis coupling is absent, while the even Willis coupling is found to be only related to the asymmetry of gain, i.e., the temperature gradient that is applied to the unit cell, already in the first-order expansion. Even and nonreciprocal Willis couplings are found of equal modulus but opposite sign, which suggests that the even coupling appears as a counter reaction to the nonreciprocal coupling and that the thermoacoustics fundamental equations describe specific forms of Willis materials. We show that both Willis couplings are almost purely imaginary at low frequencies, thus enabling a zero-group-velocity point and more importantly the opening of an amplification band at vanishing frequency. The effective parameters and scattering properties of a single unit and a 15 units system are validated against experimental results. These results pave the way for a better physical understanding of Willis couplings in nonreciprocal systems, for easing the engineering application of Willis materials, and for various applications of such systems to further control the acoustic waves at very low frequencies. Concurrently, this article also aims at promoting thermoacoustics as an excellent mean to design nonreciprocal systems.

\section{ACKNOWLEDGMENTS}

C.O., Gaëlle Poignand, and Guillaume Penelet would like to acknowledge the support of the ANR SelfiXs project (ANR-18-CE92-0001). J.-P.G., M.M., and V.R.-G. would like to acknowledge the support of the ANR-RGC METARoom project (ANR-18-CE08-0021). J.L. would like to acknowledge the support of the Research Grants Council in Hong Kong (Grant No. 16302218). D.T. acknowledges financial support through the "Ramón y Cajal" fellowship under Grant No. RYC-2016-21188 of the Ministerio de Ciencia e Innovación.

\section{APPENDIX A: EXPRESSION OF THE EFFECTIVE PROPERTIES IN THE PRESENCE OF VISCOTHERMAL LOSSES}

The viscothermal losses are accounted for in the different elements composing the unit cell through the complex functions $f_{v}$ and $f_{\kappa}$. These functions describe the viscous and thermal couplings between the oscillating gas and the surrounding solid walls [10,37] and are defined for various geometries of the waveguide channels (i.e., the waveguide itself in the case of an empty duct such as the TBT, or the pores of a porous material, as in the case of the stack or the heat exchangers). Considering $\delta_{v, \kappa}$ the frequency-dependent viscous and thermal acoustic boundary layer thicknesses given respectively by $\delta_{\kappa}=\sqrt{2 \kappa / \omega}$ and $\delta_{v}=\sqrt{\operatorname{Pr}} \delta_{\kappa}$, classical results for cylindrical channels of radius $r$ (adequate approximation for the honeycomb heat exchangers) yield

$$
f_{v, \kappa}=\frac{2}{(i+1) r / \delta_{v, \kappa}} \frac{J_{1}\left[(i+1) r / \delta_{v, \kappa}\right]}{J_{0}\left[(i+1) r / \delta_{v, \kappa}[\right.},
$$

where $J_{n}$ is the $n$th order Bessel function of the first kind and for rectangle channels of dimension $2 a \times 2 b$ (such is the case of the empty waveguide and for the stack square channels where $a=b=r_{s}$ ):

$$
f_{v, \kappa}=1-\left(\frac{8}{\pi^{2}}\right)^{2} \sum_{m} \sum_{n} F_{m, n}^{v, \kappa}
$$

with $m, n=1,3,5, \ldots$ and where

$$
F_{m, n}^{v, \kappa}=\left(m^{2} n^{2}\left\{1+i \frac{\pi^{2}}{2}\left(\frac{\delta_{v, \kappa}}{r}\right)^{2}\left[\frac{b^{2} m^{2}+a^{2} n^{2}}{(a+b)^{2}}\right]\right\}\right)^{-1}
$$

with $r=2 a b /(a+b)$ the characteristic length of the channel. The temperature dependence of the fluid (in this instance air) is accounted for through the variations of its viscosity and thermal conductivity following Ref. [38].

\section{APPENDIX B: RECOVERY PROCEDURE OF THE EFFECTIVE PARAMETERS FROM THE MEASURED REFLECTION AND TRANSMISSION COEFFICIENTS}

The two state vectors at both sides of the $L$-thick sample, whose propagation matrix is assumed to be independent of $x$ by definition, are related by

$$
\mathbf{W}(L)=\exp \left(\mathbf{A}_{e} L\right) \mathbf{W}(0)=\mathbf{V} \operatorname{diag}\left(e^{\Sigma^{ \pm} L}\right) \mathbf{V}^{-1} \mathbf{W}(0),
$$

where diag is the diagonal matrix, $\Sigma^{ \pm}$are the eigenvalues of $\mathbf{A}_{e}$, and $\mathbf{V}$ is the corresponding eigenvector matrix. For an asymmetric and nonreciprocal Willis material, the constitutive matrix of which is given by Eq. (7), $\Sigma^{ \pm}=i k^{ \pm}=i \omega \chi^{\mathrm{nr}} \pm$ $i \omega \sqrt{\left(\chi^{a}\right)^{2}+\rho / K}=i \omega \chi^{\mathrm{nr}} \pm i \omega \sigma$, and

$$
\mathbf{V}^{-1}=\frac{1}{\sqrt{2}}\left[\begin{array}{cc}
1 / K \sigma & \left(\sigma-\chi^{a}\right) / \sigma \\
-1 / K \sigma & \left(\sigma+\chi^{a}\right) / \sigma
\end{array}\right]
$$

From the expression of $\Sigma^{ \pm}$, the link with anisotropic fluid [39] becomes clear via the terms $i \omega \chi^{\mathrm{nr}}$. Introducing $Z^{ \pm}=K(\sigma \pm$ $\chi^{a}$ ), left-multiplying Eq. (B1) by $\mathbf{V}^{-1}$, and expressing the state vectors in terms of the scattering coefficients $R^{+}, T^{+}$, $R^{-}$, and $T^{-}$[see Fig. 1(b)], leads to the two following systems of equations:

$$
\begin{aligned}
{\left[\begin{array}{cc}
1 & Z^{-} \\
-1 & Z^{+}
\end{array}\right]\left(\begin{array}{c}
T^{+} \\
T^{+} / Z_{0}
\end{array}\right) } & =\left[\begin{array}{cc}
e^{\Sigma^{+} L} & Z^{-} e^{\Sigma^{+} L} \\
-e^{\Sigma^{-} d} & Z^{+} e^{\Sigma^{-} L}
\end{array}\right]\left(\begin{array}{c}
1+R^{+} \\
\left(1-R^{+}\right) / Z_{0}
\end{array}\right), \\
{\left[\begin{array}{cc}
1 & Z^{-} \\
-1 & Z^{+}
\end{array}\right]\left(\begin{array}{c}
R^{-}+1 \\
\left(R^{-}-1\right) / Z_{0}
\end{array}\right) } & =\left[\begin{array}{cc}
e^{\Sigma^{+} L} & Z^{-} e^{\Sigma^{+} L} \\
-e^{\Sigma^{-} L} & Z^{+} e^{\Sigma^{-} L}
\end{array}\right]\left(\begin{array}{c}
T^{-} \\
-T^{-} / Z_{0}
\end{array}\right),
\end{aligned}
$$


where $Z_{0}$ is the impedance (divided by the duct cross-sectional area in our case) of the surrounding fluid. Introducing $r^{ \pm}=$ $\left(Z_{0}+Z^{ \pm}\right) /\left(Z_{0}-Z^{ \pm}\right)$, these equations can be inverted to yield

$$
\begin{aligned}
r^{+} & =\frac{-\left(R^{+} R^{-}-T^{+} T^{-}+1\right) \pm \sqrt{\left(R^{+} R^{-}-T^{+} T^{-}+1\right)^{2}-4 R^{+} R^{-}}}{2 R^{+}}, \\
r^{-} & =\frac{-\left(R^{+} R^{-}-T^{+} T^{-}+1\right) \pm \sqrt{\left(R^{+} R^{-}-T^{+} T^{-}+1\right)^{2}-4 R^{+} R^{-}}}{2 R^{-}}, \\
e^{2 i \omega \sigma L} & =\frac{r^{-}\left(R^{+} r^{+}+1\right)}{R^{+}+r^{+}}, \quad e^{2 i \omega \chi^{\mathrm{nr}} L}=\frac{\left(R^{-}+r^{+}\right)\left(R^{-} r^{-}+1\right)}{\left(T^{-}\right)^{2} r^{+}} .
\end{aligned}
$$

When $T^{+}=T^{-}=T$, this set of equations collapses to that derived in [30] for reciprocal system. From these equations, $\chi_{\exp }^{a}, \chi_{\exp }^{\mathrm{nr}}, K_{\exp }$, and $\rho_{\exp }$ are subsequently recovered. Special attention must be paid to the choice of the sign in the first two equations and to folding arising when $L=N d$, $N \in \mathbb{N}$.

\section{APPENDIX C: DIRECT NUMERICAL CALCULATION OF THE EFFECTIVE PROPERTIES FROM THE TOTAL TRANSFER MATRIX}

Once the total transfer matrix $\mathbf{T}$ is calculated, it is directly assimilated to $\exp \left(\mathbf{A}_{e} d\right), \mathbf{A}_{e}$ being homogenized. From Eq. (B1), it is clear that the eigenvectors of $\mathbf{T}$ and $\mathbf{A}_{e}$ are
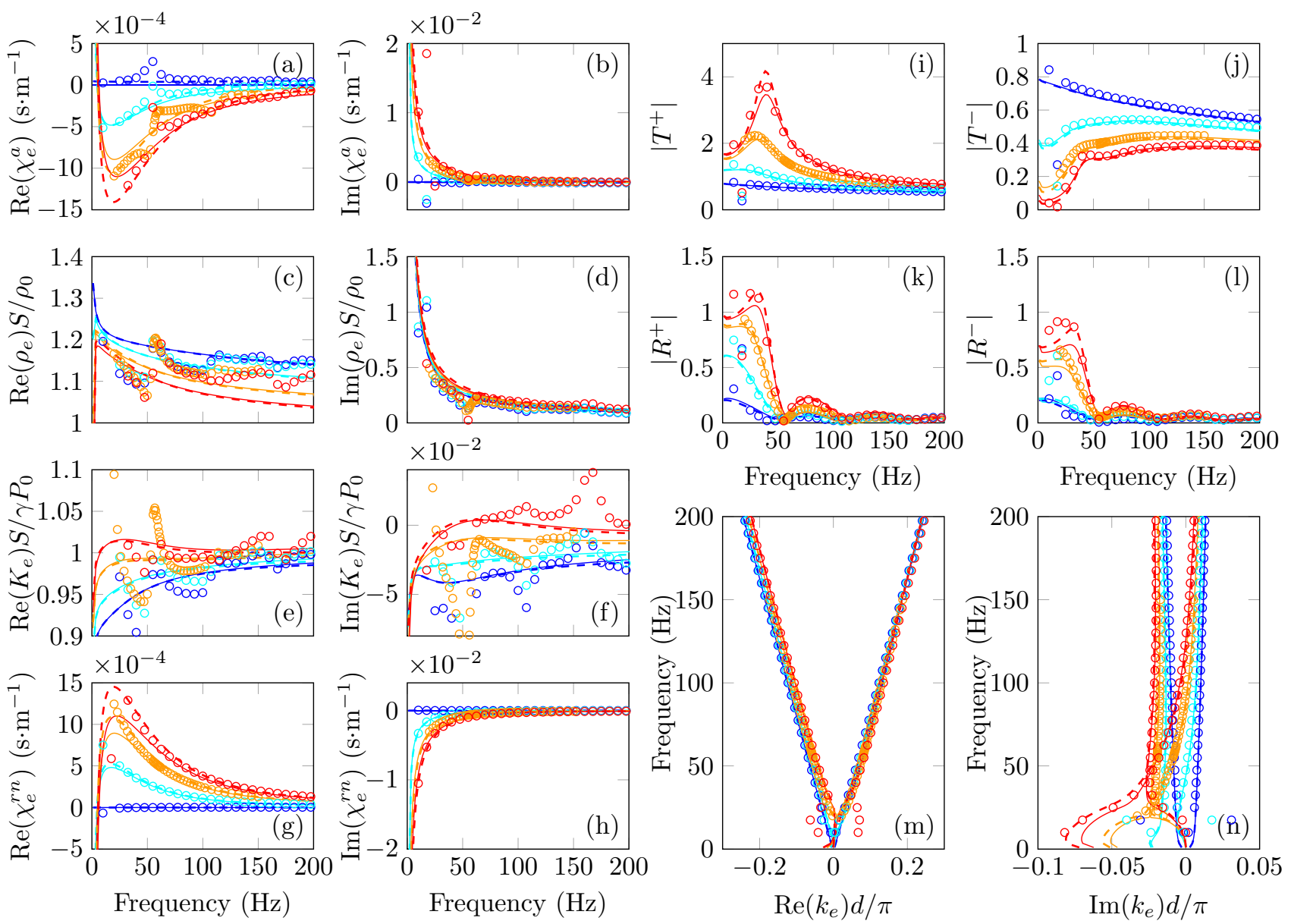

$\circ \Delta T=0 \mathrm{~K}, \circ \Delta T=25 \mathrm{~K}, \circ \Delta T=60 \mathrm{~K}, \circ \Delta T=90 \mathrm{~K}$

FIG. 4. Real and imaginary parts of the even Willis coupling parameter (a) and (b), normalized density (c) and (d), normalized bulk modulus (e) and (f), and nonreciprocal Willis coupling parameter (g) and (h) for $\Delta T=0 \mathrm{~K}$ (blue curves), $\Delta T=22 \mathrm{~K}$ (cyan curves), $\Delta T=42 \mathrm{~K}$ (green curves), $\Delta T=45 \mathrm{~K}$ (orange curves), and $\Delta T=100 \mathrm{~K}$ (red curves). Corresponding transmission and reflection coefficients along the amplification, $T^{+}$and $R^{+}$(i) and (k), and attenuation, $T^{-}$and $R^{-}(\mathrm{j})$ and (1), directions. Real and imaginary parts of the dispersion relations (m) and (n). The results depicted with solid curves are calculated with the expressions given in Eq. (11), the dashed curves represent the numerical evaluation with the transfer matrix method, and the circles depict the measurements. 
identical and that the exponential of the eigenvalues of $\mathbf{A}_{e} d$ are the eigenvalues of $\mathbf{T}$. We immediately end up with

$$
A_{e}^{\mathrm{num}}=\frac{1}{d} \mathbf{V} \operatorname{diag}\left[\log \left(\Lambda^{ \pm}\right)\right] \mathbf{V}^{-1},
$$

where $\Lambda^{ \pm}$are the eigenvalues of $\mathbf{T}$ and $\mathbf{V}$ is the associated eigenvector matrix. The four quantities $\chi_{\text {num }}^{\mathrm{nr}}, \chi_{\text {num }}^{a}, \rho_{\text {num }}$, and $K_{\text {num }}$ are subsequently evaluated.

\section{APPENDIX D: EXPERIMENTAL AND NUMERICAL VALIDATION IN CASE OF A SAMPLE COMPRISING 15 UNIT CELLS}

Experimental validation was also conducted with a sample comprising 15 unit cells, see Fig. 1. In this case, the construction of the unit cell is slightly different from that considered in Sec. IV. The stacks are the same as in the single cell system. Both ambient and hot heat exchangers are made of a $l_{1}=l_{3}=15 \mathrm{~mm}$ section of the same $1 / 8 \mathrm{in}$. aluminum honeycomb (porosity $\phi=0.945$ ), used to ensure a uniform temperature distribution over the cross section. The ambient $T_{C}$ and hot $T_{H}$ temperatures are imposed on the heat exchangers through the 1-mm-thick stainless steel walls of the waveguide, thanks to Peltier cooler modules for the cold side and polyimide heaters for the hot side, applied on each face of the waveguide. Due to the different heating system, the TBT is here considered of length $l_{4}=11.3 \mathrm{~cm}$, the overall length of the cell remaining $d=19.5 \mathrm{~cm}$. The sample total length is thus $L=15 d=292.5 \mathrm{~cm}$.

Figures 4(a)-4(h) depict the real and imaginary parts of the even and nonreciprocal Willis coupling parameters, normalized density, and bulk modulus as evaluated from the expressions given in Eq. (11) (solid curves), from the direct calculation with the transfer matrix method (see Appendix C) (dashed curves), and as measured (see Appendix B) (curves marked with circles) for $\Delta T=0 \mathrm{~K}$ (blue curves), $\Delta T=25 \mathrm{~K}$ (cyan curves), $\Delta T=60 \mathrm{~K}$ (orange curves), and $\Delta T=90 \mathrm{~K}$. Note again that the direct calculation with the transfer matrix method is conducted by discretizing the stack and the TBT (subjected to temperature gradients) in piecewise constant elements. Some drops are visible, notably on the normalized density and bulk modulus, which are due to Farby-Perot resonances, which blur the reconstruction around 60,120, and $180 \mathrm{~Hz}$. Figures 4(i)-4(1) depict the absolute values of the reflection and transmission coefficients for each incidence, i.e., that where amplification occurs $T^{+}$and $R^{+}$and that where attenuation occurs $T^{-}$and $R^{-}$. The Fabry-Perot resonances are clearly visible around 60,120 , and $180 \mathrm{~Hz}$. Note that the transfer matrix is this time calculated via $\exp \left(A_{e} L\right)$ when $A_{e}$ is used, because $L$ is no longer small with respect to the wavelength. The real and imaginary parts of the dispersion relations are depicted in Figs. 4(m) and 4(n), respectively. Slight discrepancies are again noticed for each subfigure, notably for the normalized bulk modulus and for large $\Delta T$, which are attributed to the assumption of the linear variation temperature distribution, but also to the very low frequency range of the measurements. Comments are similar to those already reported for the case of a single cell, notably with the presence of the coalescence point. Note that the amplification and attenuation are more pronounced and more visible on $T^{+}$and $T^{-}$this time and that the amplitude of the reflection coefficients are not small this time. This result validates the effective properties derived in Eq. (11) and assumption of one-dimensional system.
[1] H. Nassar, X. Xu, A. Norris, and G. Huang, J. Mech. Phys. Solids 101, 10 (2017).

[2] B.-I. Popa and S. A. Cummer, Nat. Commun. 5, 3398 (2014).

[3] A. Merkel, V. Tournat, and V. Gusev, Phys. Rev. E 90, 023206 (2014).

[4] T. Devaux, A. Cebrecos, O. Richoux, V. Pagneux, and V. Tournat, Nat. Commun. 10, 3292 (2019).

[5] R. Fleury, D. L. Sounas, C. F. Sieck, M. R. Haberman, and A. Alù, Science 343, 516 (2014).

[6] A. Merkel, M. Willatzen, and J. Christensen, Phys. Rev. Appl. 9, 034033 (2018).

[7] H. Nassar, B. Yousefzadeh, R. Fleury, M. Ruzzene, A. Alù, C. Daraio, A. N. Norris, G. Huang, and M. R. Haberman, Nat. Rev. Mater. 5, 667 (2020).

[8] T. Biwa, H. Nakamura, and H. Hyodo, Phys. Rev. Appl. 5, 064012 (2016).

[9] H. Hao, C. Scalo, and F. Semperlotti, Phys. Rev. B 104, 104303 (2021).

[10] G. W. Swift, Thermoacoustics: A Unifying Perspective for Some Engines and Refrigerators (Acoustical Society of America, Melville, NY, 2002), Chap. 4.

[11] S. L. Garrett, Am. J. Phys. 72, 11 (2004).

[12] P. H. Ceperley, J. Acoust. Soc. Am. 66, 1508 (1979).

[13] S. Backhaus and G. W. Swift, Nature (London) 399, 335 (1999).
[14] M. E. H. Tijani and S. Spoelstra, Cryogenics 48, 77 (2008).

[15] G. Poignand, P. Lotton, G. Penelet, and M. Bruneau, Acta Acust. United Acust. 97, 926 (2011).

[16] T. Yazaki, Phys. Rev. E 48, 1806 (1993).

[17] R. Delage, Y. Takayama, H. Hyodo, and T. Biwa, Chaos 29, 093108 (2019).

[18] G. Penelet and T. Biwa, Am. J. Phys. 81, 290 (2013).

[19] M. Sato, H. Hyodo, T. Biwa, and R. Delage, Chaos 30, 063130 (2020).

[20] T. Biwa, T. Takahashi, and T. Yazaki, J. Acoust. Soc. Am. 130, 3558 (2011).

[21] C. Olivier, G. Penelet, G. Poignand, J. Gilbert, and P. Lotton, Acta Acust. United Acust. 101, 941 (2015).

[22] D. Shimizu and N. Sugimoto, J. Appl. Phys. 120, 144901 (2016).

[23] G. Poignand, C. Olivier, and G. Penelet, J. Acoust. Soc. Am. 149, 1913 (2021).

[24] J. Willis, Wave Motion 3, 1 (1981).

[25] I. Lindell, A. Sihvola, S. Tretyakov, and A. J. Viitanen, Electromagnetic Waves in Chiral and Bi-isotropic Media (Artech House, Boston, 1994), pp. 1-22.

[26] C. F. Sieck, A. Alú, and M. R. Haberman, Phys. Rev. B 96, 104303 (2017).

[27] L. Quan, D. L. Sounas, and A. Alù, Phys. Rev. Lett. 123, 064301 (2019). 
[28] Y. Zhai, H.-S. Kwon, and B.-I. Popa, Phys. Rev. B 99, 220301(R) (2019).

[29] C. Cho, X. Wen, N. Park, and J. Li, Commun. Phys. 4, 82 (2021).

[30] J.-P. Groby, M. Malléjac, A. Merkel, V. Romero-García, V. Tournat, D. Torrent, and J. Li, New J. Phys. 23, 53020 (2021).

[31] K. Ding, Z. Q. Zhang, and C. T. Chan, Phys. Rev. B 92, 235310 (2015).

[32] N. Rott, Z. Angew. Math. Phys. 20, 230 (1969).

[33] M. C. Pease, Methods of Matrix Algebra (Academic, Cambridge, 1965), Chaps. 6 and 7.
[34] M. L. Munjal and A. G. Doige, J. Sound Vib. 141, 323 (1990).

[35] H. Bodén and M. Åbom, J. Acoust. Soc. Am. 79, 541 (1986).

[36] J.-P. Dalmont, J. Kergomard, and X. Meynial, C. R. Acad. Sci. Paris 309, 453 (1989).

[37] W. P. Arnott, H. E. Bass, and R. Raspet, J. Acoust. Soc. Am. 90, 3228 (1991).

[38] A. D. Pierce, Acoustics: An Introduction to Its Physical Principles and Applications (Springer International, New York, 2019), Chap. 10.1.4, p. 589.

[39] A. Terroir, L. Schwan, T. Cavalieri, V. Romero-García, G. Gabard, and J.-P. Groby, J. Appl. Phys. 125, 025114 (2019). 